\title{
On the Boundary Integral Equation Method for a Mixed Boundary Value Problem of the Biharmonic Equation
}

\author{
Fioralba Cakoni, George C. Hsiao ${ }^{\dagger}$ \\ Department of Mathematical Sciences, University of Delaware, \\ Newark, Delaware 19716, U.S.A. \\ E-mail:\{cakoni, hsiao\}@math.udel.edu \\ and \\ Wolfgang L. Wendland ${ }^{\dagger}$ \\ Institut für Angewandte Analysis und Numerische Simulation, \\ Universität Stutgart \\ Pfaffenwaldring 57, 70569 Stuttgart, Germany \\ E-mail: wendland@mathematik.uni-stuttgart.de \\ Dedicated to Heinrich Begehr on the occasion of his 65th birthday
}

\begin{abstract}
This paper is concerned with weak solution of a mixed boundary value problem for the biharmonic equation in the plane. Using Green's formula, the problem is converted into a system of Fredholm integral equations for the unknown data on different parts of the boundary. Existence and uniqueness of the solutions of the system of boundary integral equations are established in appropriate Sobolev spaces.
\end{abstract}

*The research of this author was supported in part by the Air Force Office of Scientific Research under grant F49620-02-1-0353.

$\dagger$ The research of these authors were supported in part by the German Research Foundation DFG under the Grant SFB 404 Multifield Problems in Continuum Mechanics 
AMS (MOS): 35J35, 45A05, 45F99, 35S05.

Key words: Biharmonic equation, mixed boundary value problems, boundary integral equations, variational formulations.

\section{Introduction}

In the paper [15], a mixed boundary value problem for the two-dimensional Laplace equation is considered. Using Green's formula the problem is converted into a system of Fredholm integral equations for the missing part of Cauchy data on different part of the boundary. One of these boundary integral equations has principal part of the second kind, whereas the other one is of the first kind. However, the crucial point of the approach there is that the derived system of integral equations can be interpreted as a strongly elliptic system of pseudodifferential equations. Hence it can be solved constructively by Galerkin's method.

The purpose of the present paper is to see the feasibility of extending the approach in [15] for the Laplace equation to the biharmonic equation. Clearly for the latter, it is much more involved; there is double the amount of Cauchy data and 16 boundary operators needed to be considered. However, as will be seen, the recent systematic characterization of the Calderón projector in [9] has simplified the approach in the same manner as in the case for the Laplace equation.

The paper is organized as follows: In Section 1, we formulate the mixed boundary problem and present some preliminary results for the weak solutions of the boundary value problems for the biharmonic equation. Section 2 contains the core materials for the four basic boundary integral equations. Theorems 3.3, 3.4 and 3.5 in Section 3 are the main results concerning existence and uniqueness of the system of boundary integral equations in Section 2. Finally in the last section, Section 4, we conclude the paper by a brief discussion on the regularity results of the solutions of boundary integral equations, and these can be served as the mathematical foundations for

the augmented Galerkin method in the same manner as in the case of Laplace equation (see [15]). 


\section{Formulation of the Problem}

Let $\Omega \subset \mathbb{R}^{2}$ be a bounded simply connected region with $C^{1,1}$ - boundary $\Gamma$. We assume that the boundary $\Gamma$ has a dissection $\Gamma=\Gamma_{D} \cup \Gamma_{c} \cup \Gamma_{N}$, where $\Gamma_{D}$ and $\Gamma_{N}$ are disjoint, relatively open subset of $\Gamma$, having $\Gamma_{c}$ as their common boundary points in $\Gamma$. We denote by $n=\left(n_{1}, n_{2}\right)$ the unit outward normal vector to $\Gamma$.

Now let a thin plate in elastostatic equilibrium occupy the region $\Omega$. We assume that the part $\Gamma_{D}$ of the boundary is clamped while the part $\Gamma_{N}$ is free. If we denote by $u$ the equilibrium state of the plate we obtain the following mixed boundary value problems for the biharmonic equation

$$
\begin{aligned}
& \Delta^{2} u=0 \quad \text { in } \quad \Omega \\
& u=f \quad \text { and } \quad \frac{\partial u}{\partial n}=g \quad \text { on } \quad \Gamma_{D} \\
& M u=p \quad \text { and } \quad N u=q \quad \text { on } \Gamma_{N},
\end{aligned}
$$

where the boundary operators $\left.M\right|_{\Gamma_{D}}$ and $\left.N\right|_{\Gamma_{N}}$ are the restrictions to $\Gamma_{D}$ and $\Gamma_{N}$, respectively, of the following boundary differential operators

$$
M u=\nu \Delta u+(1-\nu) M_{0} u
$$

and

$$
N u=-\frac{\partial}{\partial n} \Delta u-(1-\nu) \frac{\partial}{\partial s} N_{0} u \text {. }
$$

Here $\nu$ is the Poisson ratio, a real constant, and in applications (especially in the theory of elasticity) we have $0 \leq \nu<1$. The normal and tangential derivatives are given by

$$
\frac{\partial}{\partial n}=n_{1} \frac{\partial}{\partial x_{1}}+n_{2} \frac{\partial}{\partial x_{2}} \quad \text { and } \quad \frac{\partial}{\partial s}=-n_{1} \frac{\partial}{\partial x_{2}}+n_{2} \frac{\partial}{\partial x_{1}},
$$

while the boundary operators $M_{0} u$ and $N_{0} u$ are defined by

$$
M_{0} u:=\frac{\partial^{2} u}{\partial x_{1}^{2}} n_{1}^{2}+2 \frac{\partial^{2} u}{\partial x_{1} \partial x_{2}} n_{1} n_{2}+\frac{\partial^{2} u}{\partial x_{2}^{2}} n_{2}^{2}
$$

and

$$
N_{0} u:=-\left\{\left(\frac{\partial^{2} u}{\partial x_{1}^{2}}-\frac{\partial^{2} u}{\partial x_{2}^{2}}\right) n_{1} n_{2}-\frac{\partial^{2} u}{\partial x_{1} \partial x_{2}}\left(n_{1}^{2}-n_{2}^{2}\right)\right\} .
$$

Physically, $M u$ is the bending moment and $N u$ is the transverse force consisting of the shear force and twisting moment [2]. The mixed conditions (2) 
and (3) may be interpreted that the plate is clamped on $\Gamma_{D}$ and has a free edge on $\Gamma_{N}$. We are interested in the weak solution of the mixed boundary value problem, (1), (2), and (3).

Our solution space for the biharmonic equation (1) is the standard Sobolev space $H^{2}(\Omega)$ of distributions that are square integrable and have square integrable derivatives up to the second order. We first observe that since the boundary $\Gamma$ is $C^{1,1}$, the trace spaces $H^{\frac{3}{2}}(\Gamma)$ and $H^{\frac{1}{2}}(\Gamma)$ are well defined [3] and moreover for $u \in H^{2}(\Omega)$ we have that $\left.u\right|_{\Gamma} \in H^{\frac{3}{2}}(\Gamma)$ and $\left.\frac{\partial u}{\partial n}\right|_{\Gamma} \in H^{\frac{1}{2}}(\Gamma)$. To discuss the boundary value problem for (1), it is best to begin with the Green formula for (1) in $\Omega$. By using integration by parts, one obtains

$$
\int_{\Omega}\left(\Delta^{2} u\right) v d x=a(u, v)-\int_{\Gamma}\left[(M u) \frac{\partial v}{\partial n}+(N u) v\right] d s
$$

for smooth functions, where the bilinear form $a(u, v)$ is defined by

$$
\begin{aligned}
a_{\Omega}(u, v) & :=\int_{\Omega} \nu \Delta u \Delta v d x \\
& +\int_{\Omega}(1-\nu)\left(\frac{\partial^{2} u}{\partial x_{1}^{2}} \frac{\partial^{2} v}{\partial x_{1}^{2}}+2 \frac{\partial^{2} u}{\partial x_{1} \partial x_{2}} \frac{\partial^{2} v}{\partial x_{1} \partial x_{2}}+\frac{\partial^{2} u}{\partial x_{2}^{2}} \frac{\partial^{2} v}{\partial x_{2}^{2}}\right) d x
\end{aligned}
$$

We note that the bilinear form in $(7)$ is well defined for functions in $H^{2}(\Omega)$. Now let $u \in H^{2}\left(\Omega, \Delta^{2}\right)$ where

$$
H^{2}\left(\Omega, \Delta^{2}\right):=\left\{u \in H^{2}(\Omega): \quad \Delta^{2} u \in \widetilde{H}^{-2}(\Omega)\right\}
$$

with $\widetilde{H}^{-2}(\Omega)$ denoting the dual space of $H^{2}(\Omega)$ and choose $v \in H^{2}(\Omega)$. Then the above Green formula holds and by a duality argument one shows that $M u \in H^{-\frac{1}{2}}(\Gamma)$ and $N u \in H^{-\frac{3}{2}}(\Gamma)$ are well defined, where $H^{-\frac{1}{2}}(\Gamma)$ and $H^{-\frac{3}{2}}(\Gamma)$ are the dual spaces of $H^{\frac{1}{2}}(\Gamma)$ and $H^{\frac{3}{2}}(\Gamma)$, respectively.

For later use we recall the following classical result by Agmon [1].

Lemma 2.1 Assume that $-3<\nu<1$. Then the bilinear form $a(\cdot, \cdot)$ given by (7) satisfies a Garding inequality in the form

$$
a(v, v)_{\Omega} \geq c_{0}\|v\|_{H^{2}(\Omega)}^{2}-\lambda_{0}\|v\|_{L^{2}(\Omega)}^{2}
$$

for all $v \in H^{2}(\Omega)$, where $c_{0}>0$ and $\lambda_{0} \geq 0$ are constant. 
In order to formulate the mixed boundary value problem (1)-(3) more precisely we need to define the following trace spaces on an open arc $\Gamma_{0} \subset \Gamma$. For any $s \in \mathbb{R}, s \geq 0$, we define

$$
\begin{aligned}
H^{s}\left(\Gamma_{0}\right) & :=\left\{\left.u\right|_{\Gamma_{0}}: u \in H^{s}(\Gamma)\right\} \\
\widetilde{H}^{s}\left(\Gamma_{0}\right) & :=\left\{u \in H^{s}(\Gamma): \operatorname{supp} u \subseteq \bar{\Gamma}_{0}\right\}
\end{aligned}
$$

(see, e.g., [15]). For $s>0$ we denote by $H^{-s}\left(\Gamma_{0}\right)$ the dual space of $\widetilde{H}^{s}\left(\Gamma_{0}\right)$ and by $\widetilde{H}^{-s}\left(\Gamma_{0}\right)$ the dual space of $H^{s}\left(\Gamma_{0}\right)$ with $L^{2}\left(\Gamma_{0}\right)$ as the pivot space. Note that $\widetilde{H}^{-s}\left(\Gamma_{0}\right)$ can also be identify with the space of distributions in $H^{-s}(\Gamma)$ supported in $\bar{\Gamma}_{0}$. The following chain of continuous embeddings holds

$$
\widetilde{H}^{s}\left(\Gamma_{0}\right) \subset H^{s}\left(\Gamma_{0}\right) \subset L^{2}\left(\Gamma_{0}\right) \subset \widetilde{H}^{-s}\left(\Gamma_{0}\right) \subset H^{-s}\left(\Gamma_{0}\right) \quad s>0 .
$$

Now we are ready to formulate precisely the mixed boundary value problem for the biharmonic equation: Given $f \in H^{\frac{3}{2}}\left(\Gamma_{D}\right), g \in H^{\frac{1}{2}}\left(\Gamma_{D}\right), p \in$ $H^{-\frac{1}{2}}\left(\Gamma_{N}\right)$, and $q \in H^{-\frac{3}{2}}\left(\Gamma_{N}\right)$, find $u \in H^{2}(\Omega)$ that satisfies (1)-(3). We will refer to this problem as (MBP).

Theorem 2.2 The mixed boundary value problem (MBP) has at most one solution for $0 \leq \nu \leq 1$.

Proof. Let $u$ be the solution of (MBP) with $f=g=p=q=0$. Then an application of Green's formula to $u$ and $\bar{u}$ yields

$$
\int_{\Omega}\left[\nu|\Delta u|^{2}+(1-\nu)\left(\left|\frac{\partial^{2} u}{\partial x_{1}^{2}}\right|^{2}+2\left|\frac{\partial^{2} u}{\partial x_{1} \partial x_{2}}\right|^{2}+\left|\frac{\partial^{2} u}{\partial x_{2}^{2}}\right|^{2}\right)\right] d x=0 .
$$

Hence for $0 \leq \nu<1$ we have $\frac{\partial^{2} u}{\partial x_{1}^{2}}=\frac{\partial^{2} u}{\partial x_{2}^{2}}=0$ in $\Omega$ which implies $u=$ $a x_{1}+b x_{2}+c$ and form the boundary conditions $\left.u\right|_{\Gamma_{D}}=\partial u /\left.\partial n\right|_{\Gamma_{D}}=0$ we conclude that $u=0$ in $\Omega$.

In the case of $\nu=1$ we obtain that $\Delta u=0$ in $\Omega$ and $u=\frac{\partial u}{\partial n}=0$ on $\Gamma_{D}$. Now let $B_{\rho}$ be a ball of radius $\rho$ with center on $\Gamma_{D}$ such that $\bar{B}_{\rho} \cap \Gamma_{N}=0$ and define $v=u$ in $\Omega \cap B_{\rho}, v=0$ in $\left(\mathbb{R}^{2} \backslash \bar{\Omega}\right) \cap B_{\rho}$. Then $v$ satisfies $\Delta v=0$ in $B_{\rho}$ and hence real-analytic in $B_{\rho}$. We can now conclude that $u \equiv 0$ in $B_{\rho}$ and thus $u \equiv 0$ in $\Omega$. 


\section{Boundary Integral Equations}

In order to prove the existence of a solution to (MBP) and provide a solution formula we will reformulate the problem as a system of boundary integral equations of the first kind. We start with the Green representation formula of a weak solution in $H^{2}(\Omega)[9]$

$$
u(x)=\mathcal{V}(M u, N u)(x)-\mathcal{W}\left(u, \frac{\partial u}{\partial n}\right)(x), \quad x \in \Omega
$$

in terms of simple- and double-layer potentials. Here

$$
\mathcal{V}: H^{-\frac{1}{2}}(\Gamma) \times H^{-\frac{3}{2}}(\Gamma) \longrightarrow H^{2}(\Omega), \quad \text { and } \quad \mathcal{W}: H^{\frac{3}{2}}(\Gamma) \times H^{\frac{1}{2}}(\Gamma) \longrightarrow H^{2}(\Omega)
$$

are continuous operators defined by

$$
\begin{aligned}
\mathcal{V}\left(\sigma_{1}, \sigma_{2}\right)(x): & =\int_{\Gamma}\left\{E(x, y) \sigma_{2}(y)+\frac{\partial E(x, y)}{\partial n_{y}} \sigma_{1}\right\} d s_{y}, \quad x \in \mathbb{R}^{2} \backslash \Gamma, \\
\mathcal{W}\left(\phi_{1}, \phi_{2}\right)(x): & =\int_{\Gamma}\left\{M_{y} E(x, y) \phi_{2}(y)+N_{y} E(x, y) \phi_{1}(y)\right\} d s_{y}, \quad x \in \mathbb{R}^{2} \backslash \Gamma
\end{aligned}
$$

where

$$
E(x, y):=\frac{1}{8 \pi}|x-y|^{2} \log |x-y|
$$

is the fundamental solution of the biharmonic equation. Letting $x \rightarrow \Gamma$ from inside $\Omega$, and following the standard procedure in potential theory involving jump relations, we obtain the following integral equations on $\Gamma$,

$$
\begin{aligned}
\left.u(x)\right|_{\Gamma}:= & \int_{\Gamma}\left\{E(x, y) N u(y)+\frac{\partial E(x, y)}{\partial n_{y}} M u(y)\right\} d s_{y} \\
- & \int_{\Gamma} M_{y} E(x, y) \frac{\partial u}{\partial n}(y) d s_{y}+\left[\frac{1}{2} u(x)-\int_{\Gamma} N_{y} E(x, y) u(y) d s_{y}\right] \\
\left.\frac{\partial u}{\partial n}(x)\right|_{\Gamma}: & =\int_{\Gamma}\left\{\frac{\partial E(x, y)}{\partial n_{x}} N u(y)+\frac{\partial^{2} E(x, y)}{\partial n_{x} \partial n_{y}} M u(y)\right\} d s_{y} \\
& +\left[\frac{1}{2} \frac{\partial u}{\partial n}(x)-\int_{\Gamma} \frac{\partial}{\partial n_{x}} M_{y} E(x, y) \frac{\partial u}{\partial n_{y}}(y) d s_{y}\right] \\
& -\int_{\Gamma} \frac{\partial}{\partial n_{x}} N_{y} E(x, y) u(y) d s_{y}
\end{aligned}
$$




$$
\begin{aligned}
\left.M u(x)\right|_{\Gamma}: & =\int_{\Gamma} M_{x} E(x, y) N u(y) d s_{y}+\left[\frac{1}{2} M u(x)-\int_{\Gamma} M_{x} \frac{\partial E(x, y)}{\partial n_{y}} M u(y) d s_{y}\right] \\
& -\int_{\Gamma}\left\{M_{x} M_{y} E(x, y) \frac{\partial u}{\partial n_{y}}(y)+M_{x} N_{y} E(x, y) u(y)\right\} d s_{y} \\
\left.N(x)\right|_{\Gamma}:= & {[11) } \\
& -\int_{\Gamma}\left\{u(x)+\int_{\Gamma} N_{x} E(x, y) N u(y) d s_{y}\right]+\int_{\Gamma} N_{x} \frac{\partial E(x, y)}{\partial n_{y}} M u(y) d s_{y}
\end{aligned}
$$

In order to understand the mapping properties of the above 16 boundary integral operators we may rewrite (9)-(12) in the form

$$
\left[\begin{array}{c}
u \\
\frac{\partial u}{\partial n} \\
M u \\
N u
\end{array}\right]_{\left.\right|_{\Gamma}}=\left[\begin{array}{cccc}
\frac{1}{2} I-K_{11} & V_{12} & V_{13} & V_{14} \\
D_{21} & \frac{1}{2} I+K_{22} & V_{23} & V_{24} \\
D_{31} & D_{32} & \frac{1}{2} I-K_{33} & V_{34} \\
D_{41} & D_{42} & D_{43} & \frac{1}{2} I+K_{44}
\end{array}\right]\left[\begin{array}{c}
u \\
\frac{\partial u}{\partial n} \\
M u \\
N u
\end{array}\right]_{\left.\right|_{\Gamma}}
$$

where the operators are defined in an obvious manner. This matrix of integral operators is the corresponding Calderón projector for the biharmonic equation with respect to domain $\Omega$ and will be denoted by $C_{\Omega}:=$ $\left(\left(C_{\Omega}^{i, j}\right)\right)_{4 \times 4}$. This Calderón projector in fact consists of pseudodifferential operators on $\Gamma$ and has been studied in details in [9]. In particular it maps $H^{\frac{3}{2}}(\Gamma) \times H^{\frac{1}{2}}(\Gamma) \times H^{-\frac{1}{2}}(\Gamma) \times H^{-\frac{3}{2}}(\Gamma)$ into itself continuously. The mapping properties of each of the operators appearing in $C_{\Omega}$ can be easily obtained from its principal symbol as

$$
\operatorname{Ord}\left(C_{\Omega}\right):=\left[\begin{array}{rrcc}
0 & -1 & -3 & -3 \\
+1 & 0 & -1 & -3 \\
+1 & +1 & 0 & -1 \\
+3 & +1 & +1 & 0
\end{array}\right]
$$

We note that in particular the order of each operator $C_{\Omega}^{i, j}$ in the matrix $C_{\Omega}$ can be computed from the difference of its index $i-j$ (or $i-j-1$, when $|i-j|=2$ ). The operators of negative order are smoothing operators while the operators of positive order are singular and the absolute value of the 
order shows how much regularity we gain or loose by mapping. For instance the operator $C_{\Omega}^{13}=V_{1,3}$ defined by

$$
\left(V_{13} \varphi\right)(x):=\int_{\Gamma} \frac{\partial E(x, y)}{\partial n_{y}} \varphi(y) d s_{y}
$$

is of order -2 (in fact in this case is of order $-2-1$ ) and hence maps continuously for $\Gamma \in C^{\infty}$ from $H^{-\frac{1}{2}}(\Gamma)$ to $H^{\frac{3}{2}}(\Gamma)\left(H^{\frac{5}{2}}(\Gamma)\right)$, while the operator $C_{\Omega}^{42}=D_{42}$,

$$
\left(D_{42} \varphi\right)(x):=\int_{\Gamma} N_{x} M_{y} E(x, y) \varphi(y) d s
$$

is of order 2 (or rather $2-1$ ) and maps from $H^{\frac{1}{2}}(\Gamma)$ to $H^{-\frac{3}{2}}(\Gamma)\left(H^{-\frac{1}{2}}(\Gamma)\right)$ continuously.

Now we return to the mixed boundary value problem (1)-(3). We denote by $\tilde{f} \in H^{\frac{3}{2}}(\Gamma), \tilde{g} \in H^{\frac{1}{2}}(\Gamma), \tilde{p} \in H^{-\frac{1}{2}}(\Gamma)$ and $\tilde{q} \in H^{-\frac{3}{2}}(\Gamma)$ bounded extensions to the whole of $\Gamma$ of the corresponding boundary data $f, g, p$ and $q$. Then we write

$$
\begin{aligned}
\left.u\right|_{\Gamma} & =\phi_{N}+\tilde{f} & & \left.\frac{\partial u}{\partial n}\right|_{\Gamma}=\psi_{N}+\tilde{g} \\
\left.M u\right|_{\Gamma} & =\sigma_{D}+\tilde{p} & & \left.N u\right|_{\Gamma}=\tau_{D}+\tilde{q} .
\end{aligned}
$$

Obviously $\phi_{N} \in \widetilde{H}^{\frac{3}{2}}\left(\Gamma_{N}\right), \psi_{N} \in \widetilde{H}^{\frac{1}{2}}\left(\Gamma_{N}\right), \sigma_{D} \in \widetilde{H}^{-\frac{1}{2}}\left(\Gamma_{D}\right)$ and $\tau_{D} \in \widetilde{H}^{-\frac{3}{2}}\left(\Gamma_{D}\right)$ since $\phi_{N}=\psi_{N}=0$ on $\Gamma_{D}$ and $\sigma_{D}=\tau_{D}=0$ on $\Gamma_{N}$.

By now restricting (9) and (10) to $\Gamma_{D}$ and (11) and (12) to $\Gamma_{N}$ we obtain the following system of boundary integral equations of the first kind for $\phi_{N}, \psi_{N}, \sigma_{D}, \tau_{D}$

$$
\begin{gathered}
\int_{\Gamma_{D}} E(x, y) \tau_{D}(y) d s+\int_{\Gamma_{D}} \frac{\partial E(x, y)}{\partial n_{y}} \sigma_{D}(y) d s-\int_{\Gamma_{N}} M_{y} E(x, y) \psi_{N}(y) d s \\
-\int_{\Gamma_{N}} N_{y} E(x, y) \phi_{N}(y) d s=F_{1}(x), \quad x \in \Gamma_{D} \\
\int_{\Gamma_{D}} \frac{\partial E(x, y)}{\partial n_{y}} \tau_{D}(y) d s+\int_{\Gamma_{D}} \frac{\partial^{2} E(x, y)}{\partial n_{x} \partial n_{y}} \sigma_{D}(y) d s-\int_{\Gamma_{N}} \frac{\partial}{\partial n_{x}} M_{y} E(x, y) \psi_{N}(y) d s \\
-\int_{\Gamma_{N}} \frac{\partial}{\partial n_{x}} N_{y} E(x, y) \phi_{N}(y) d s=F_{2}(x), \quad x \in \Gamma_{D}
\end{gathered}
$$




$$
\begin{gathered}
\int_{\Gamma_{D}} M_{x} E(x, y) \tau_{D}(y) d s+\int_{\Gamma_{D}} M_{x} \frac{\partial E(x, y)}{\partial n_{y}} \sigma_{D}(y) d s-\int_{\Gamma_{N}} M_{x} M_{y} E(x, y) \psi_{N}(y) d s \\
-\int_{\Gamma_{N}} M_{x} N_{y} E(x, y) \phi_{N}(y) d s=F_{3}(x), \quad x \in \Gamma_{N} \\
\int_{\Gamma_{D}} N_{x} E(x, y) \tau_{D}(y) d s+\int_{\Gamma_{D}} N_{x} \frac{\partial E(x, y)}{\partial n_{y}} \sigma_{D}(y) d s-\int_{\Gamma_{N}} N_{x} M_{y} E(x, y) \psi_{N}(y) d s \\
-\int_{\Gamma_{N}} N_{x} N_{y} E(x, y) \phi_{N}(y) d s=F_{4}(x), \quad x \in \Gamma_{N}
\end{gathered}
$$

where

$$
\begin{aligned}
F_{1}(x)= & -\int_{\Gamma} E(x, y) \tilde{q}(y) d s-\int_{\Gamma} \frac{\partial E(x, y)}{\partial n_{y}} \tilde{p}(y) d s \\
+ & \int_{\Gamma} M_{y} E(x, y) \tilde{g}(y) d s+\left[\frac{1}{2} \tilde{f}(x)+\int_{\Gamma} N_{y} E(x, y) \tilde{f}(y) d s\right], \quad x \in \Gamma_{D} \\
F_{2}(x)= & -\int_{\Gamma} \frac{\partial E(x, y)}{\partial n_{y}} \tilde{q}(y) d s-\int_{\Gamma_{D}} \frac{\partial^{2} E(x, y)}{\partial n_{x} \partial n_{y}} \tilde{p}(y) d s \\
+ & {\left[\frac{1}{2} \tilde{g}(x)+\int_{\Gamma} \frac{\partial}{\partial n_{x}} M_{y} E(x, y) \tilde{g}(y) d s\right]+\int_{\Gamma} \frac{\partial}{\partial n_{x}} N_{y} E(x, y) \tilde{f}(y) d s, \quad x \in \Gamma_{D} } \\
F_{3}(x)= & -\int_{\Gamma} M_{x} E(x, y) \tilde{q}(y) d s\left[\frac{1}{2} \tilde{p}(x)-\int_{\Gamma} M_{x} \frac{\partial E(x, y)}{\partial n_{y}} \tilde{p}(y) d s\right] \\
& +\int_{\Gamma} M_{x} M_{y} E(x, y) \tilde{g}(y) d s+\int_{\Gamma} M_{x} N_{y} E(x, y) \tilde{f}(y) d s, \quad x \in \Gamma_{N}
\end{aligned}
$$




$$
\begin{aligned}
F_{4}(x) & =\left[\frac{1}{2} \tilde{q}(x)-\int_{\Gamma} N_{x} E(x, y) \tilde{q}(y) d s\right]-\int_{\Gamma} N_{x} \frac{\partial E(x, y)}{\partial n_{y}} \tilde{p}(y) d s \\
& +\int_{\Gamma} N_{x} M_{y} E(x, y) \tilde{g}(y) d s+\int_{\Gamma} N_{x} N_{y} E(x, y) \tilde{f}(y) d s, \quad x \in \Gamma_{N}
\end{aligned}
$$

The system of equations (15)-(18) can be written in a matrix form as follows

$$
\mathcal{A}\left[\begin{array}{c}
\tau_{D} \\
\sigma_{D} \\
\psi_{N} \\
\phi_{N}
\end{array}\right]:=\left[\begin{array}{cccc}
V_{14}^{D D} & V_{13}^{D D} & V_{12}^{D N} & -K_{11}^{D N} \\
V_{24}^{D D} & V_{23}^{D D} & K_{22}^{D N} & D_{21}^{D N} \\
V_{34}^{N D} & -K_{33}^{N D} & D_{32}^{N N} & D_{31}^{N N} \\
K_{44}^{N D} & D_{43}^{N D} & D_{42}^{N N} & D_{41}^{N N}
\end{array}\right]\left[\begin{array}{c}
\tau_{D} \\
\sigma_{D} \\
\psi_{N} \\
\phi_{N}
\end{array}\right]=\mathbf{F}
$$

with $\mathbf{F}=\left[F_{1}, F_{2}, F_{3}, F_{4}\right]^{\top}$. Here $V_{i j}, D_{i, j}, K_{i i} i=1 \ldots 4, j=1 \ldots 4$ are the operators that appear in the Calderón operator, and $V_{24}^{D N}$ stands for the operator $V_{24}$ applied to a function with support in $\Gamma_{N}$ and evaluated on $\Gamma_{D}$, with analogous definitions for the other operators. From the mapping properties of the Calderón operator, one can see that the operator $\mathcal{A}$ defines a continuous mapping $\mathcal{A}: \mathcal{H} \rightarrow \mathcal{H}^{*}$ with $\mathcal{H}:=\widetilde{H}^{-\frac{3}{2}}\left(\Gamma_{D}\right) \times \widetilde{H}^{-\frac{1}{2}}\left(\Gamma_{D}\right) \times$ $\widetilde{H}^{\frac{1}{2}}\left(\Gamma_{N}\right) \times \widetilde{H}^{\frac{3}{2}}\left(\Gamma_{N}\right)$ and $\mathcal{H}^{*}:=H^{\frac{3}{2}}\left(\Gamma_{D}\right) \times H^{\frac{1}{2}}\left(\Gamma_{D}\right) \times H^{-\frac{1}{2}}\left(\Gamma_{N}\right) \times H^{-\frac{3}{2}}\left(\Gamma_{N}\right)$ the dual space of $\mathcal{H}$.

We remark that if $\phi_{N}, \psi_{N}, \sigma_{D}, \tau_{D}$ satisfy (19) then, after defining $u, \frac{\partial u}{\partial n}$, $M u, N u$ on $\Gamma$ by (13) and (14), the representation formula (8) gives a solution to (MBP) which from Theorem 2.2 is the unique solution. Hence we need to study the solvability of the system of integral equations of the first kind (19). For this purpose, let us first introduce the matrix operator $V: H^{-\frac{3}{2}}(\Gamma) \times$ $H^{-\frac{1}{2}}(\Gamma) \rightarrow H^{\frac{3}{2}}(\Gamma) \times H^{\frac{1}{2}}(\Gamma)$ defined by

$$
V:=\left[\begin{array}{ll}
V_{14} & V_{13} \\
V_{24} & V_{23}
\end{array}\right]
$$

Then the following lemma holds.

Lemma 3.1 There exists a compact operator $C_{V}: H^{-\frac{3}{2}}(\Gamma) \times H^{-\frac{1}{2}}(\Gamma) \rightarrow$ $H^{\frac{3}{2}}(\Gamma) \times H^{\frac{1}{2}}(\Gamma)$ such that

$$
\left\langle\left(V+C_{V}\right) \Theta, \Theta\right\rangle \geq C\|\Theta\|_{H^{-3 / 2}(\Gamma) \times H^{-1 / 2}(\Gamma)}^{2} \quad \text { for } \quad \Theta \in H^{-\frac{3}{2}}(\Gamma) \times H^{-\frac{1}{2}}(\Gamma)
$$

where the bracket $\langle\cdot, \cdot\rangle$ denotes the $L_{2}(\Gamma)$-duality pairing between $H^{-\frac{3}{2}}(\Gamma) \times$ $H^{-\frac{1}{2}}(\Gamma)$ and $H^{\frac{3}{2}}(\Gamma) \times H^{\frac{1}{2}}(\Gamma)$. 
Proof. The proof follows Hsiao and Wendland [7] and Costabel and Wendland [4]. For any $\Theta=\left(\theta_{1}, \theta_{2}\right) \in H^{-\frac{3}{2}}(\Gamma) \times H^{-\frac{1}{2}}(\Gamma)$, let

$$
u(x)=\int_{\Gamma}\left\{E(x, y) \theta_{1}(y)+\frac{\partial E(x, y)}{\partial n_{y}} \theta_{2}\right\} d s_{y}, \quad x \in \mathbb{R}^{2} \backslash \Gamma .
$$

Then $u \in H^{2}\left(\Omega, \Delta^{2}\right), u \in H_{l o c}^{2}\left(\Omega_{c}, \Delta^{2}\right)$ where $\Omega_{c}:=\mathbb{R}^{2} \backslash \bar{\Omega}$. Moreover (9)-(12) yield

$$
[u]_{\Gamma}=0, \quad\left[\frac{\partial u}{\partial n}\right]_{\Gamma}=0, \quad[M u]_{\Gamma}=\theta_{2}, \quad[N u]_{\Gamma}=\theta_{1}
$$

where $[\cdot]_{\Gamma}$ denotes the jump across the boundary $\Gamma$. Next we introduce a fixed $C_{0}^{\infty}\left(\mathbb{R}^{2}\right)$ cut-off function $\chi$ with $\left.\chi\right|_{\Omega}=1$. Hence from the jump properties, (9)-(12) and the Green formula (6) we can write

$$
\langle V \Theta, \Theta\rangle=\int_{\Gamma}\left(\frac{\partial u}{\partial n}[M u]+u[N u]\right) d s=a_{\Omega}(u, u)+a_{\Omega_{c}}(\chi u, \chi u),
$$

where $a_{\Omega}(\cdot, \cdot)$ and $a_{\Omega_{c}}(\cdot, \cdot)$ are the bilinear forms $(7)$ corresponding to $\Omega$ and $\Omega_{c}$. Note that $a_{\Omega_{c}}(\chi u, \chi u)$ is well defined since $\chi u$ has compact support. Then the result follows from Lemma 2.1, the fact that $H^{2}$ is compactly imbedded in $L^{2}$ and the Riesz representation theorem. It is important to notice that $\chi u=u$ in a neighborhood of $\Gamma$ and therefore a Garding inequality of the type of Lemma 2.1 holds also for $a_{\Omega_{c}}(\cdot, \cdot)$ since the integrals containing $\chi$ are simply compact perturbations (for details see the proof of Theorem 3.7 in [8] for the case of the Laplace equation).

Next, let $D: H^{\frac{1}{2}}(\Gamma) \times H^{\frac{3}{2}}(\Gamma) \rightarrow H^{-\frac{1}{2}}(\Gamma) \times H^{-\frac{3}{2}}(\Gamma)$ be the continuous mapping defined by

$$
D:=\left[\begin{array}{cc}
D_{32} & D_{31} \\
D_{42} & D_{41}
\end{array}\right]
$$

Similarly, we have the following lemma.

Lemma 3.2 There exists a compact operator $C_{D}: H^{\frac{1}{2}}(\Gamma) \times H^{\frac{3}{2}}(\Gamma) \rightarrow H^{-\frac{1}{2}}(\Gamma) \times$ $H^{-\frac{3}{2}}(\Gamma)$ such that

$$
\left\langle\left(D+C_{D}\right) \Psi, \Psi\right\rangle \geq C\|\Psi\|_{H^{1 / 2}(\Gamma) \times H^{3 / 2}(\Gamma)}^{2} \quad \text { for } \quad \Psi \in H^{\frac{1}{2}}(\Gamma) \times H^{\frac{3}{2}}(\Gamma)
$$

where the bracket $\langle\cdot, \cdot\rangle$ denotes the $L_{2}(\Gamma)$-duality pairing between $H^{\frac{1}{2}}(\Gamma) \times$ $H^{\frac{3}{2}}(\Gamma)$ and $H^{-\frac{1}{2}}(\Gamma) \times H^{-\frac{3}{2}}(\Gamma)$. 
Proof. For any $\Psi=\left(\psi_{1}, \psi_{2}\right) \in H^{\frac{1}{2}}(\Gamma) \times H^{\frac{3}{2}}(\Gamma)$, let

$$
u(x)=\int_{\Gamma}\left\{M_{y} E(x, y) \psi_{1}(y)+N_{y} E(x, y) \psi_{2}\right\} d s_{y} \quad x \in \mathbb{R}^{2} \backslash \Gamma .
$$

Then $u \in H^{2}\left(\Omega, \Delta^{2}\right), u \in H_{l o c}^{2}\left(\Omega_{c}, \Delta^{2}\right)$ and from (9)-(12) we have that

$$
[u]_{\Gamma}=\psi_{2}, \quad\left[\frac{\partial u}{\partial n}\right]_{\Gamma}=\psi_{1}, \quad[M u]_{\Gamma}=0, \quad[N u]_{\Gamma}=0
$$

Then we can write

$$
\begin{aligned}
\langle D \Psi, \Psi\rangle & =\int_{\Gamma}\left(N_{x}[u]+M_{x} u\left[\frac{\partial u}{\partial n}\right]\right) d s \\
& =a_{\Omega}(u, u)+a_{\Omega_{c}}(\chi u, \chi u)
\end{aligned}
$$

and the result is obtain by the same argument as in Lemma 3.1 where $\chi$ is the cut-off function introduced in Lemma 3.1.

Theorem 3.3 Let $\mathcal{H}:=\widetilde{H}^{-\frac{3}{2}}\left(\Gamma_{D}\right) \times \widetilde{H}^{-\frac{1}{2}}\left(\Gamma_{D}\right) \times \widetilde{H}^{\frac{1}{2}}\left(\Gamma_{N}\right) \times \widetilde{H}^{\frac{3}{2}}\left(\Gamma_{N}\right)$ and its dual $\mathcal{H}^{*}:=H^{\frac{3}{2}}\left(\Gamma_{D}\right) \times H^{\frac{1}{2}}\left(\Gamma_{D}\right) \times H^{-\frac{1}{2}}\left(\Gamma_{N}\right) \times H^{-\frac{3}{2}}\left(\Gamma_{N}\right)$. Then the operator $\mathcal{A}: \mathcal{H} \rightarrow \mathcal{H}^{*}$ is Fredholm with index zero.

Proof. From Lemma 3.1 and 3.2, let $V_{0}=V+C_{V}$ and $D_{0}=D+C_{D}$, where $V$ and $D$ are defined by (20) and (21). Then we know that $V_{0}$ and $D_{0}$ are bounded below and positive. Since $\Xi:=\left(\tau_{D}, \sigma_{D}, \psi_{N}, \phi_{N}\right) \in \widetilde{H}^{-\frac{3}{2}}\left(\Gamma_{D}\right) \times$ $\widetilde{H}^{-\frac{1}{2}}\left(\Gamma_{D}\right) \times \widetilde{H}^{\frac{1}{2}}\left(\Gamma_{N}\right) \times \widetilde{H}^{\frac{3}{2}}\left(\Gamma_{N}\right)$ it can be extended by zero to a function $\tilde{\Xi}:=\left(\tilde{\tau}_{D}, \tilde{\sigma}_{D}, \tilde{\psi}_{N}, \tilde{\phi}_{N}\right)$ in $H^{-\frac{3}{2}}(\Gamma) \times H^{-\frac{1}{2}}(\Gamma) \times H^{\frac{1}{2}}(\Gamma) \times H^{\frac{3}{2}}(\Gamma)$. Therefore we can write $\mathcal{A}$ in the form

$$
\mathcal{A}=\mathcal{A}_{0}+\mathcal{C}_{\mathcal{A}}:=\left[\begin{array}{cr}
V_{0} & M_{D N} \\
M_{N D} & D_{0}
\end{array}\right]+\mathcal{C}_{\mathcal{A}}
$$

where $\mathcal{C}_{\mathcal{A}}$ is compact, and

$$
M_{D N}:=\left[\begin{array}{cc}
V_{12}^{D N} & -K_{11}^{D N} \\
K_{22}^{D N} & D_{21}^{D N}
\end{array}\right] \quad \text { and } \quad M_{N D}:=\left[\begin{array}{cc}
V_{34}^{N D} & -K_{33}^{N D} \\
K_{44}^{N D} & D_{43}^{D N}
\end{array}\right]
$$


Furthermore from (15)-(18) we have

$$
\begin{aligned}
\left\langle V_{12}^{D N} \psi_{N}, \tau_{D}\right\rangle & =\int_{\Gamma_{D}} \tau_{D}(x) \int_{\Gamma_{N}} M_{y} E(x, y) \psi_{N}(y) d s(y) d s(x) \\
& =\int_{\Gamma} \tilde{\tau}_{D}(x) \int_{\Gamma} M_{y} E(x, y) \tilde{\psi}_{N}(y) d s(y) d s(x) \\
& =-\int_{\Gamma} \tilde{\psi}_{N}(y) \int_{\Gamma} M_{x} E(x, y) \tilde{\tau}_{D}(x) d s(x) d s(y) \\
& =-\int_{\Gamma_{N}} \psi_{N}(y) \int_{\Gamma_{D}} M_{x} E(x, y) \tau_{D}(x) d s(x) d s(y) \\
& =-\left\langle V_{34}^{N D} \tau_{D}, \psi_{N}\right\rangle .
\end{aligned}
$$

In the same manner one can show that

$$
\begin{aligned}
\left\langle K_{11}^{D N} \phi_{N}, \tau_{D}\right\rangle & =\left\langle K_{44}^{N D} \tau_{D}, \phi_{N}\right\rangle \\
\left\langle K_{22}^{D N} \psi_{N}, \sigma_{D}\right\rangle & =\left\langle K_{33}^{N D} \sigma_{D}, \psi_{N}\right\rangle
\end{aligned}
$$

and

$$
\left\langle D_{21}^{D N} \phi_{N}, \sigma_{D}\right\rangle=-\left\langle D_{43}^{N D} \sigma_{D}, \phi_{N}\right\rangle \text {. }
$$

Finally combining these equations and using Lemmas (3.1) and (3.2) we obtain

$$
\begin{aligned}
\left\langle\mathcal{A}_{0} \Xi, \Xi\right\rangle_{\mathcal{H}, \mathcal{H}^{*}}= & \left\langle V_{0}\left(\tau_{D}, \sigma_{D}\right),\left(\tau_{D}, \sigma_{D}\right)\right\rangle+\left\langle D_{0}\left(\psi_{N}, \phi_{N}\right),\left(\psi_{N}, \phi_{N}\right)\right\rangle \\
& \geq c_{1}\left\|\left(\tau_{D}, \sigma_{D}\right)\right\|_{H^{-3 / 2} \times H^{-1 / 2}}^{2}+c_{2}\left\|\left(\psi_{N}, \phi_{N}\right)\right\|_{H^{1 / 2} \times H^{3 / 2}}^{2} \\
& \geq c\|\Xi\|_{\mathcal{H}}^{2}
\end{aligned}
$$

for any $\Xi \in \mathcal{H}$ where $c>0$ is a constant. Hence $\mathcal{A}$ is a Fredholm operator with index zero. In particular the uniqueness of (19) implies the existence of the solution to (19).

The next theorem establishes the uniqueness of (19).

Theorem 3.4 The kernel of the operator $\mathcal{A}: \mathcal{H} \rightarrow \mathcal{H}^{*}$ is zero.

Let $\Xi:=\left(\tau_{D}, \sigma_{D}, \psi_{N}, \phi_{N}\right) \in \tilde{H}^{-\frac{3}{2}}\left(\Gamma_{D}\right) \times \widetilde{H}^{-\frac{1}{2}}\left(\Gamma_{D}\right) \times \widetilde{H}^{\frac{1}{2}}\left(\Gamma_{N}\right) \times \widetilde{H}^{\frac{3}{2}}\left(\Gamma_{N}\right)$ be a solution to the homogeneous equation $\mathcal{A} \Xi=0$, and $\tilde{\Xi}:=\left(\tilde{\tau}_{D}, \tilde{\sigma}_{D}, \tilde{\psi}_{N}, \tilde{\phi}_{N}\right)$ 
in $H^{-\frac{3}{2}}(\Gamma) \times H^{-\frac{1}{2}}(\Gamma) \times H^{\frac{1}{2}}(\Gamma) \times H^{\frac{3}{2}}(\Gamma)$ be the extension by zero. The the potential $w$ defined by

$$
\begin{aligned}
w(x) & =\mathcal{V}\left(\tilde{\sigma}_{D}, \tilde{\tau}_{D}\right)(x)-\mathcal{W}\left(\tilde{\phi}_{N}, \tilde{\psi}_{N}\right)(x) \\
& =\int_{\Gamma}\left\{E(x, y) \tilde{\tau}_{D}+\frac{\partial E(x, y)}{\partial n_{y}} \tilde{\sigma}_{D}\right\} d s_{y} \\
& -\int_{\Gamma}\left\{M_{y} E(x, y) \tilde{\psi}_{N}(y)+N_{y} E(x, y) \tilde{\phi}_{N}(y)\right\} d s_{y}
\end{aligned}
$$

is in $H^{2}\left(\Omega, \Delta^{2}\right)$ and $H_{l o c}^{2}\left(\Omega_{c}\right)$ and satisfies the biharmonic equation. Now let $x \rightarrow \Gamma$ from inside $\Omega$, using the jump relations (9)-(10) we obtain:

$$
\begin{aligned}
\left.w\right|_{\Gamma} & =V_{14} \tilde{\tau}_{D}+V_{13} \tilde{\sigma}_{D}+V_{12} \tilde{\psi}_{N}+\left[\frac{1}{2} \tilde{\phi}_{N}-K_{11} \tilde{\phi}_{N}\right] \\
\left.\frac{\partial w}{\partial n}\right|_{\Gamma} & =V_{24} \tilde{\tau}_{D}+V_{23} \tilde{\sigma}_{D}+\left[\frac{1}{2} \tilde{\psi}_{N}+K_{22} \tilde{\psi}_{N}\right]+D_{21} \tilde{\phi}_{N} \\
\left.M w\right|_{\Gamma} & =V_{34} \tilde{\tau}_{D}+\left[\frac{1}{2} \tilde{\sigma}_{D}-K_{33} \tilde{\sigma}_{D}\right]+D_{32} \tilde{\psi}_{N}+D_{31} \tilde{\phi}_{N} \\
\left.N w\right|_{\Gamma} & =\left[\frac{1}{2} \tilde{\tau}_{D}+K_{44} \tilde{\tau}_{D}\right]+D_{43} \tilde{\sigma}_{D}+D_{42} \tilde{\psi}_{N}+D_{41} \tilde{\phi}_{N} .
\end{aligned}
$$

Using the fact that $\operatorname{supp} \sigma_{D}, \operatorname{supp} \tau_{D}$ are in $\Gamma_{D}$ and $\operatorname{supp} \phi_{N}, \operatorname{supp} \psi_{N}$ are in $\Gamma_{N}$, the integral equation $\mathcal{A} \Xi=0$ implies that

$$
\left.w\right|_{\Gamma_{D}}=0,\left.\quad \frac{\partial w}{\partial n}\right|_{\Gamma_{D}}=0,\left.\quad M w\right|_{\Gamma_{N}}=0,\left.\quad N w\right|_{\Gamma_{N}}=0 .
$$

The latter means that (22) is a weak solution to homogeneous interior mixed boundary value for biharmonic equation, and hence, from Theorem $2.2, w=$ 0 in $\Omega$.

Now, using Green's representation formula (8) for $w \in H^{2}(\Omega)$ we have

$$
0=\mathcal{V}(M w, N w)(x)-\mathcal{W}\left(w, \frac{\partial w}{\partial n}\right)(x), \quad x \in \Omega
$$


whence from the above boundary conditions $\Xi:=\left(\tau_{D}, \sigma_{D}, \psi_{N}, \phi_{N}\right) \in \widetilde{H}^{-\frac{3}{2}}\left(\Gamma_{D}\right) \times$ $\widetilde{H}^{-\frac{1}{2}}\left(\Gamma_{D}\right) \times \widetilde{H}^{\frac{1}{2}}\left(\Gamma_{N}\right) \times \widetilde{H}^{\frac{3}{2}}\left(\Gamma_{N}\right)$ in the kernel of $\mathcal{A}$ satisfies

$$
\begin{aligned}
0 & =\int_{\Gamma_{D}}\left\{E(x, y) \tau_{D}(y)+\frac{\partial E(x, y)}{\partial n_{y}} \sigma_{D}\right\} d s_{y} \\
& -\int_{\Gamma_{N}}\left\{M_{y} E(x, y) \psi_{N}(y)+N_{y} E(x, y) \phi_{N}(y)\right\} d s_{y}, \quad x \in \Omega .
\end{aligned}
$$

Let us denote by

$$
h(x)=\int_{\Gamma_{N}}\left\{M_{y} E(x, y) \psi_{N}(y)+N_{y} E(x, y) \phi_{N}(y)\right\} d s_{y} .
$$

From the mapping properties of the potentials, we have that $h \in H^{2}\left(\mathbb{R}^{2} \backslash \Gamma\right)$ and from the asymptotic behavior of the biharmonic double layer potential $h(x)=O(r)$ as $r=|x| \rightarrow \infty$. Therefore from the uniqueness of the exterior Dirichlet problem for the biharmonic equation with the required growth conditions (see [9]), we can represent $h$ in the form of a simple potential

$$
h(x)=\int_{\Gamma}\left\{E(x, y) \kappa(y)+\frac{\partial E(x, y)}{\partial n_{y}} \xi(y)\right\} d s_{y}
$$

with densities $\xi \in H^{-\frac{1}{2}}(\Gamma)$ and $\kappa \in H^{-\frac{3}{2}}(\Gamma)$ subject to the constraints

$$
\int_{\Gamma} \kappa(y) d s_{y}=0 \quad \int_{\Gamma} y_{i} \kappa(y)+n_{i} \xi(y) d s_{y}=0 \quad i=1,2 .
$$

The jump relations imply that

$$
\xi=M h^{+}-M h^{-}, \quad \kappa=N h^{+}-N h^{-} \quad \text { on } \Gamma
$$

where the $\mp$ signs correspond to the interior and the exterior domain, respectively. From the definition of $h$ given by (25) we see that $\xi=0$ and $\kappa=0$ on $\Gamma_{D}$, i.e. their support is included in $\bar{\Gamma}_{N}$. Inserting (26) into (24) gives

$$
\mathcal{V}(\xi, \kappa)=-\mathcal{V}\left(\tilde{\sigma}_{D}, \tilde{\tau}_{D}\right) \quad \text { in } \Omega
$$

which implies that

$$
\xi=-\tilde{\sigma}_{D} \quad \text { and } \quad \kappa=-\tilde{\tau}_{D} \quad \text { on } \Gamma
$$


are the unique solutions because of (27) (see [9]), where $\tilde{\tau}_{D} \in H^{-\frac{3}{2}}(\Gamma)$ and $\tilde{\sigma}_{D} \in H^{-\frac{1}{2}}(\Gamma)$ are the extension by zero to the whole $\Gamma$ of $\tau_{D}$ and $\sigma_{D}$. But since the support of $\tilde{\sigma}_{D}$ and $\tilde{\tau}_{D}$ intersect the support of $\xi$ and $\kappa$ only in a finite set of boundary points we can conclude that

$$
\tilde{\sigma}_{D}=\tilde{\tau}_{D}=\xi=\kappa=0 .
$$

Hence

$$
h(x)=\int_{\Gamma_{N}}\left\{M_{y} E(x, y) \psi_{N}(y)+N_{y} E(x, y) \phi_{N}(y)\right\} d s_{y}=0 \quad \text { in } \Omega .
$$

The jump relation and the fact that $\mathcal{A} \tilde{\Xi}=0, \tilde{\Xi}:=\left(\tilde{\tau}_{D}, \tilde{\sigma}_{D}, \tilde{\psi}_{N}, \tilde{\phi}_{N}\right)=$ $\left(0,0, \tilde{\psi}_{N}, \tilde{\phi}_{N}\right)$ now imply

$$
\begin{aligned}
0 & =V_{12} \tilde{\psi}_{N}+\left(\frac{1}{2} \tilde{\phi}_{N}-K_{11} \tilde{\phi}_{N}\right)=\frac{1}{2} \tilde{\phi}_{N} \\
0 & =\left(\frac{1}{2} \tilde{\psi}_{N}+K_{22} \tilde{\psi}_{N}\right)+D_{21} \tilde{\phi}_{N}=\frac{1}{2} \tilde{\psi}_{N} .
\end{aligned}
$$

So, we have shown that the kernel of $\mathcal{A}$ is zero, which proves the theorem.

Summarizing the above analysis we have proved the following result.

Theorem 3.5 Assume that $0<\nu<1$ and let $f \in H^{\frac{3}{2}}\left(\Gamma_{D}\right), g \in H^{\frac{1}{2}}\left(\Gamma_{D}\right)$, $p \in H^{-\frac{1}{2}}\left(\Gamma_{N}\right)$, and $q \in H^{-\frac{3}{2}}\left(\Gamma_{N}\right)$ be given. Then the mixed boundary value problem (1)-(3) has a weak solution in $H^{2}\left(\Omega, \Delta^{2}\right)$. Moreover the solution satisfies the estimate

$$
\|u\|_{H^{2}(\Omega)} \leq c\left(\|f\|_{H^{3 / 2}\left(\Gamma_{D}\right)}+\|g\|_{H^{1 / 2}\left(\Gamma_{D}\right)}+\|p\|_{H^{-1 / 2}\left(\Gamma_{N}\right)}+\|q\|_{H^{-3 / 2}\left(\Gamma_{N}\right)}\right)
$$

with c a positive constant.

Remark In the same way one can treat other types of mixed boundary value problems for the biharmonic equation that correspond to different physical combinations of the boundary conditions.

\section{Remarks on the Regularity of the Solution}

To conclude the paper, we now discuss briefly the regularity of the solution of the Boundary Integral Equation. In general the change of the boundary 
condition causes the solution of the mixed boundary value problem for the biharmonic equation (1)-(3) to be singular in the neighborhood of the boundary interface points in $\Gamma_{c}$ even for $C^{\infty}$-data. From Kondratjev's theory (see e.g. [11]) we know that in suitable polar coordinates with the origin at a point in $\Gamma_{c}$ (where the boundary conditions change) the most singular part of the solution is in general of the form $r^{3 / 2} F(\theta)$.

However our main concern in this section is to discuss the singularity of the solution of the boundary integral equation of the first kind $\mathcal{A} \Xi=\mathbf{F}$ near the interface point. Ultimately our solution formula leads to a boundary element method, and an understanding of the singularities of the solution of the integral equation is necessary to improve the numerical scheme. In particular, one map employ an augmented Galerkin method where the approximating space is enriched by singular functions that have the same singularity as the solution of the integral equation (see [12] for the Laplace equation).

For sufficiently smooth boundary it can be shown [2], [9] that the Calderón operator $C_{\Omega}$ maps $H^{\frac{3}{2}+s}(\Gamma) \times H^{\frac{1}{2}+s}(\Gamma) \times H^{-\frac{1}{2}+s}(\Gamma) \times H^{-\frac{3}{2}+s}(\Gamma)$ into itself for $s>0$. Hence, assuming smooth boundary and smooth boundary data $f, g, p, q$ the right hand side $\mathbf{F}$ of the integral equation $\mathcal{A} \Xi=\mathbf{F}$ is sufficiently smooth. In order to obtain the local behavior of the solution near the interface point one may use the localization procedure of Eskin [10] and apply near the interface point in $\Gamma_{c}$ the Wiener-Hopf technique which is based on the factorization of the homogeneous elliptic symbols in half line. Applications of this technique to the boundary integral equations for singular boundary value problem for Helmholtz and Maxwell equations are very well developed by Stephan [13] and [14] and Costabel and Stephan [5].

First we note that one needs to treat only the case when $\Omega$ coincides with $y>0, \Gamma_{D}$ with $R_{-}:=\{x: x<0\}$ and $\Gamma_{N}$ with $R_{+}:=\{x: x>0\}$ (here $(0,0)$ is the interface point). It suffices to consider the following two $2 \times 2$ systems

$$
p_{-} V \Theta=\mathbf{h}_{1} \quad \text { on } R_{-} \quad \text { and } \quad p_{+} D \Psi=\mathbf{h}_{2} \quad \text { on } R_{+}
$$

where $p_{( \pm)}$denotes the restriction to the half line $R_{( \pm)}$, the operators $V$ and $D$ are given by (20) and (21) respectively, and $\mathbf{h}_{1}$ and $\mathbf{h}_{\mathbf{2}}$ are the respective restrictions of $F_{1}, F_{2}$ and $F_{3}, F_{4}$. Roughly speaking, the original integral equation is separated into two first kind integral equation on half line. Note that $p_{-} V \Theta=\mathbf{h}_{1} \quad$ on $\quad R_{-}$corresponds to the crack problem on $\Gamma_{D}$ with Dirichlet boundary conditions while $p_{+} D \Psi=\mathbf{h}_{2}$ on $R_{+}$corresponds to 
the crack problem on $\Gamma_{N}$ with Neumann boundary condition. By using one dimensional Fourier transform, straightforward but long calculations [9] show that the above pseudodifferential operators are essentially similar to those in [13], [14], whence are suitable to perform a Wiener-Hopf factorization. For instance, the principal symbol $\sigma(V)$ of $V$ is given by

$$
\sigma(V)=\left(\begin{array}{cc}
\frac{1}{4\left|\xi_{1}\right|^{3}} & 0 \\
0 & -\frac{1}{4\left|\xi_{1}\right|}
\end{array}\right) \quad \text { for } \quad\left(\xi_{1}, 0\right) \in \partial \mathbb{R}_{+}^{2},
$$

where $\xi_{1}$ is the dual variable of $x$ of the Fourier transform.

In the following we only state regularity results providing that the boundary $\Gamma$ is sufficiently smooth. Let $P_{1} \in \Gamma_{c}$ be a point where the boundary conditions change and let $f \in H^{\frac{5}{2}+\eta}\left(\Gamma_{D}\right), g \in H^{\frac{3}{2}+\eta}\left(\Gamma_{D}\right), p \in H^{\frac{1}{2}+\eta}\left(\Gamma_{N}\right)$ and $q \in H^{-\frac{1}{2}+\eta}\left(\Gamma_{N}\right)$ where $0<\eta<\frac{1}{2}-\epsilon$ with some $\epsilon>0$. Then the solution $\Xi=\left(\tau_{D}, \sigma_{D}, \psi_{N}, \phi_{N}\right)$ of the integral equation

$$
\mathcal{A} \Xi=\mathbf{F}
$$

is such that

$$
\begin{aligned}
& \tau_{D}=c_{1} \rho^{-3 / 2} \chi(\rho)+\tau^{r e g} \quad \text { on } \Gamma_{D} \quad \tau^{r e g} \in \widetilde{H}^{-\frac{1}{2}+\eta^{\prime}}\left(\Gamma_{D}\right) \\
& \sigma_{D}=c_{2} \rho^{-1 / 2} \chi(\rho)+\sigma^{r e g} \quad \text { on } \Gamma_{D} \quad \sigma^{r e g} \in \widetilde{H}^{\frac{1}{2}+\eta^{\prime}}\left(\Gamma_{D}\right) \\
& \psi=c_{3} \rho^{1 / 2} \chi(\rho)+\psi^{\text {reg }} \quad \text { on } \Gamma_{N} \quad \psi^{r e g} \in \widetilde{H}^{\frac{3}{2}+\eta^{\prime}}\left(\Gamma_{N}\right) \\
& \phi=c_{4} \rho^{3 / 2} \chi(\rho)+\phi^{r e g} \quad \text { on } \Gamma_{N} \quad \phi^{r e g} \in \widetilde{H}^{\frac{5}{2}+\eta^{\prime}}\left(\Gamma_{N}\right)
\end{aligned}
$$

where $0<\eta^{\prime}<\eta, \rho$ is the distance to $P_{1}, \chi$ is a $C^{\infty}$ cut-off function with $\chi=1$ for $|\rho|<\frac{1}{2}$ and $\chi=0$ for $|\rho|>1$ and $c_{1}, c_{2}, c_{3}, c_{4}$ are constants. The above expression provides a decomposition into regular and singular parts of the solution of the boundary integral equations. In particular, even for $C^{\infty}$ boundary data we have that the solution exhibits singularities around the interface points in $\Gamma_{c}$ which needs to be taken into consideration in the boundary element approximations if higher order convergence is desired.

\section{References}

[1] S. Agmon, Lectures on Elliptic Boundary Value Problems D. Van Nostrand Co., Inc., 1965. 
[2] G. Chen and J. Zhou, Boundary Element Methods Academic Press, 1992.

[3] M. Costabel, Boundary integral operators on Lipschitz domains: elementary results, SIAM J. Math. Anal. 19 (1988), 613-626.

[4] M. Costabel, E. Stephan and W.L. Wendland, On boundary integral equations of the first kind for the bi-Laplacian in a polygonal plane domain, Ann. Scuola Norm. Sup. Pisa Cl. Sci. (4) 10, (1986) 197-241.

[5] M. Costabel and E. Stephan, An improved boundary element Galerkin method for three dimensional crack problems, Int. Eqs. Operator Theory. 10, (1987) 467-505.

[6] M. Costabel and W. L. Wendland, Strong ellipticity of boundary integral operators, J. Reine Angew. Math. 372, (1986) 34-63.

[7] G. C. Hsiao and W.L. Wendland, A finite element method for some integral equations of the first kind, J. Math. Anal. Appl. 34, (1977) $1-19$.

[8] G. C. Hsiao and W. L. Wendland, Boundary element methods: foundation and error analysis in Encyclopedia of Computational Machanics E.Stein, R.deBrost and T.J.R.Hughes, John Wiley \& Sons 2004.

[9] G.C. Hsiao and W.L. Wendland, Boundary Integral Equations: Variational Methods, Springer-Verlag: Heidelberg, to appear.

[10] G. I. Eskin Boundary Problems for Elliptic Pseudo-Differential Operators, Transl. of Math. Mon., American Math. Soc., Providence, 1981.

[11] S.A. Nazarov and B.A. Plamenevksy, Elliptic Problems in Domains with Piecewise Smooth Boundaries, Walter de Gruyer \& Co., Berlin, 1994.

[12] E. Stephan and W.L Wendland, An augmented Galerkin procedure for the boundary integral method applied to mixed boundary value problems. Appl. Numer. Math. 1, no. 2 (1985), 121-143.

[13] E. Stephan, Boundary equations for mixed boundary value problems, screen and transmission problems in $\mathbb{R}^{3}$. Habilitationsschrift (THDPreprint 848 Darmstadt) (1984). 
[14] E. Stephan, Boundary equations for mixed boundary value problems in $\mathbb{R}^{3}$. Math. Nachr. 184 (1987), 21-53.

[15] W.L. Wendland, E. Stephan and G.C. Hsiao On the integral equation method for the plane mixed boundary value problem of Laplacian, Math. Methods Appl. Sci. 1 (1979), 265-321. 